\title{
Cerebrospinal fluid and blood biomarkers of status epilepticus
}

Aurélie Hanin, PharmD ${ }^{1}$

Virginie Lambrecq, $\mathrm{MD}^{1,2,3}$

Jérôme Alexandre Denis, $\mathrm{PhD}^{3,4}$

Françoise Imbert-Bismut, $\mathrm{PhD}^{4}$

Benoît Rucheton, PharmD ${ }^{4}$

Foudil Lamari, $\mathrm{PhD}^{4}$

Dominique Bonnefont-Rousselot, PharmD ${ }^{4,5}$

Sophie Demeret, $\mathrm{MD}^{2}$

Vincent Navarro, $\mathrm{MD}^{1,2,3}$

${ }^{1}$ Brain and Spine Institute, ICM, Inserm U 1127, CNRS UMR 7225, Sorbonne Université, F75013, Paris, France

${ }^{2}$ AP-HP, GH Pitié-Salpêtrière-Charles Foix, Neurology Department, Epilepsy Unit (VL, VN) and Neuro-Intensive care Unit (SD), Paris 75013, France

${ }^{3}$ Sorbonne Université, 75005 Paris, France

${ }^{4}$ AP-HP, GH Pitié-Salpêtrière-Charles Foix, Department of Endocrine and Oncological

Biochemistry (J.AD) and Metabolic Biochemistry (BR, DBR, FI, FL), Paris 75013, France

${ }^{5}$ Paris University, UTCBS, U 1022 Inserm, UMR 8258 CNRS, Paris, France

Correspondance to: Vincent Navarro

Affiliation: Brain and Spine Institute, ICM, Inserm U 1127, CNRS UMR 7225, Sorbonne

Université, F-75013, Paris, France and AP-HP, GH Pitié-Salpêtrière-Charles Foix, Epilepsy

Unit

Adress/City/Country: 47-83 Boulevard de l'Hôpital, Paris 75013, France

Telephone: 0142161811

Email: vincent.navarro@aphp.fr

Text pages: 32 / Words: 5310 / References: 75 / Table: 1 / Figures: 4 


\section{Summary}

Status epilepticus is a condition resulting either from the failure of the mechanisms responsible for seizure termination or from the initiation of mechanisms which lead to abnormally prolonged seizures and require urgent administration of antiepileptic drugs. Refractory status epilepticus requires anesthesics drugs and may lead to brain injury with molecular and cellular alterations (e.g. inflammation, neuronal and astroglial injury) that could induce neurological sequels and further development of epilepsy. Outcome scores based on demographic, clinical and EEG condition are available, allowing to predict the risk of mortality, but the severity of brain injury in survivors is poorly evaluated. New biomarkers are needed in order to predict with higher accuracy the outcome of patients admitted with status in an intensive care unit.

Here, we summarize the findings of studies from patients and animal models of status epilepticus. Specific protein markers can be detected in the cerebrospinal fluid and the blood. One of the first described markers of neuronal death is the neuron-specific enolase. Gliosis resulting from inflammatory responses after status can be detected through the increase of S100-beta, or some cytokines, like the High Mobility Group Box 1. Other proteins, like progranuline may reflect the neuroprotective mechanisms resulting from the brain adaptation to excitotoxicity. These new biomarkers aim to prospectively identify the severity and development of disability, and subsequent epilepsy of patients suffering from status.

We discuss the advantages and disadvantages of each biomarker, by evaluating their brain specificity, stability in the fluids and sensitivity to external interferences, such as haemolysis. Finally, we emphasize the need of further development and validation of such biomarkers in order to better assess patients with severe status epilepticus. 
Abbreviations: BBB = blood-brain barrier; GFAP = glial fibrillary acidic protein; HMGB1 = High Mobility Group Box 1; NSE = neuron specific enolase; PGRN = progranulin; SE = status epilepticus

Key words: epilepsy, prognosis, critical care, neuronal injury, inflammation

\section{Introduction}

Epilepsy is one of the most common neurological disorders wich affects approximately $0.7 \%$ of the population ${ }^{1}$. Status epilepticus (SE), is a condition resulting either from the failure of the mechanisms responsible for seizure termination or from the initiation of mechanisms which lead to abnormally prolonged seizures ${ }^{2}$. Most SE can be treated by adequate antiepileptic drugs, and such patients generally recover well. Nevertheless, in around $25 \%$ of cases, SE is refractory and requires anesthetic drugs ${ }^{3}$. For these patients, SE may lead to brain injury with cellular and molecular alterations (e.g. inflammation, or neuronal and astroglial injury) that could induce subsequent irreversible neurological sequels (Fig. 1), further development of epilepsy, with a mortality rate ranging from $7 \%$ to $39 \%{ }^{4}$. Difference in mortality depends on the semiology of SE (increased mortality in non-convulsive SE with coma), the level of consciousness (increased case fatality rate in patients with impaired consciousness), and age (increased mortality in subjects over 65 years) ${ }^{4,5}$.

New biomarkers combined with clinical data, EEG (Fig. 2) and MRI (Fig. 1), may help clinicians to predict SE outcome with more accuracy ${ }^{6,7}$. Cerebrospinal fluid (CSF) biomarkers have already been established in neurological diseases such as Alzheimer's disease, Parkinson's disease, multiple sclerosis or in the traumatic brain ${ }^{8-11}$. In SE, even if CSF biomarkers have been proposed, none have been yet validated in clinical use to diagnose SE or predict its clinical outcome. 
In this review, we provide an overview of the current research on promising biomarkers of SE in CSF and blood (Table 1, Fig. 3). We present arguments for further development and validation of such biomarkers, and for their use in assessing patients with SE.

\section{How biomarkers can help management of SE?}

There is a large number of possible clinical presentations of SE (i.e. focal SE, absence SE, generalized convulsive SE, myoclonic SE, non convulsive SE) ${ }^{12}$. The diagnosis of convulsive SE relies mainly on clinical assessment. On the other hand, the diagnosis of non-convulsive SE relies on EEG recording (Fig. 2) which requires specialized clinical staff and equipment that are not available to all medical units at all times. While clinical semiology and EEG are the best features for a positive diagnosis of SE, non-EEG biomarkers would be of great help, in particular for the diagnosis of the non-convulsive SE.

Clinical SE prognosis factors, such as generalized convulsive status, patients of advanced age, and long duration of SE have been correlated with higher mortality rate ${ }^{4}$. But the most important outcome factor is the underlying aetiology: patients with post-anoxic SE, acute brain injury have a higher mortality rate than patients with a SE related to antiepileptic drug withdrawal. Nevertheless, consequences of SE from a single type of aetiology are heterogenous. Outcome scores based on demographic, clinical and EEG condition are available ${ }^{13}$. However, they predict the bad outcome (i.e. death) but the severity of brain injury in survivors is poorly evaluated. New biomarkers are needed to predict with a higher accuracy the outcome of patients admitted with SE in an intensive care unit. Even if some biochemical biomarkers are not specific to SE, we assume that they can be combined with clinical and paraclinical data (EEG and MRI) to help clinicians predict SE outcome. 
Biomarkers able to identify SE aetiology may be very useful in clinical practice. There are now guidelines to identify the aetiology of SE in acute phase ${ }^{14}$. The Status Epilepticus Etiology Identification Tool (SEEIT) is based on semiology, head computer tomography scan, and on first level blood tests (blood glucose, basic metabolic panel, anti-epileptic drug levels if appropriate,...). According to these first results, this tool aims to propose more specific blood or CSF tests ${ }^{14}$. We can speculate on our future ability to isolate specific biomarkers of acute causes of SE. Similarly, we may identify biomarkers such as cytokines levels or specific interleukines, that could suggest infectious or auto-immune SE.

Biomarkers may also be used to identify specific etiologies requiring individualized treatments. Thereby, a recent use of IL6 monoclonal antibodies has been specifically proposed in NORSE and IL-1 receptor antagonist in FIRES ${ }^{15}$.

\section{Physiologic responses to status epilepticus and late consequences}

A good fluid biomarker is a molecule: (i) whose measurements in fluid provides high sensitivity and high specificity tests; (ii) whose biochemical assessment is easy, quick and reliable and (iii) which is stable in the body fluids and demonstrates links to molecular and cellular alterations that occur in SE.

During SE, physiologic responses occur first and then pathological late consequences can lead to neuronal death and subsequent epilepsy.

During convulsive SE, some physiologic changes occur outside of the brain; many of them may worsen the acute brain injury. They include: (i) hyperthermia 16; (ii) immediate and marked increase in plasma catecholamine concentrations, that may provoke increased cerebral blood flow ${ }^{17}$; (iii) hypoglycemia; (iv) marked and rapidly developing acidosis, which could be beneficial or harmful for the brain as the acidosis is respectively modest or significant ${ }^{18}$. 
Our understanding of the late consequences of SE and epileptogenic process comes largely from animal models of induced SE. Epileptogenesis refers to the phenomenon in which an initial brain insult (e.g. traumatic brain injury, stroke, or SE) triggers a cascade of molecular and cellular changes that eventually culminate in the occurrence of spontaneous seizures. Five leading changes can be identified after a SE. (i) One major change is the neuronal death. The origin of this acute neurodegeneration mainly comes from excitotoxic processes. It was described in the hippocampus, amygdala as well as in many extratemporal areas including the thalamus ${ }^{19}$. The therapies used to reduce neurodegeneration decrease the behavioral consequences after SE in rats ${ }^{20}$. Neuronal loss can be evaluated by the blood concentration of specific proteins such as neuronal markers ${ }^{21}$. (ii) Increased neurogenesis could be detected within few days and remained up to four weeks after the onset of SE ${ }^{22}$. This process may reflect the brain adaptation to the neuronal death ${ }^{23}$, and can be evaluated by the levels of mediators involved in neuronal growth factor, such as progranulin ${ }^{24}$. Nevertheless, neurogenesis is limited to some brain areas, especially the hippocampus. (iii) Gliosis results from inflammatory responses ${ }^{25,26}$. An increased expression of glial cell markers (glial fibrillary acidic protein) and proteins secreted by glial cells (e.g. cytokines, chemokines) was reported, as a consequence of cytokines release during excitotoxic processes and glial cell activation related to cleaning processes after neuronal death. Gliosis may modify ionic homeostasis and further enhance neuronal excitability. (iv) Blood-brain barrier breakdown may contribute to the development of epileptogenesis via a direct mechanism (influx of potassium) or via the leakage of serum proteins in the brain causing glial cells activation as well as inflammation and impaired potassium buffering ${ }^{27}$. (v) Axonal injury can be evaluated by measuring tau protein in the cerebrospinal fluid. 


\section{Potential cerebrospinal fluid and blood biomarkers of status epilepticus}

Cerebrospinal fluid (CSF) is a source of promising biomarkers. CSF is in close contact with the extracellular space of the brain compared to the plasma or the serum, and its composition closely reflects biochemical changes that occur in the brain ${ }^{8,9}$. CSF biomarkers have already been established in neurological diseases such as Alzheimer's disease, Parkinson's disease, multiple sclerosis or in the traumatic brain injury ${ }^{8-11}$.

In SE, even if CSF biomarkers have been proposed, none has yet been validated for clinical use. Some proteins that are highly expressed in the brain (e.g. S100-B, NSE, and cytokines) can be detected in the serum, even if in low concentrations. It will be very useful to benefit from blood biomarkers of SE because a lumbar puncture is invasive, not systematically performed in routine clinical care for patients in SE, and can not be easily repeated, for daysor weeks-long kinetic studies and patients follow-up.

Here we present an overview of biomarkers relating to the pathophysiology of SE.

\section{Acidosis}

A marked and rapidly developing acidosis is noted in patients with SE and in animal models of SE. In humans, a study conducted in twenty-nine adult patients with generalized SE of various etiologies showed an increase of CSF lactate for all SE patients with a mean at $3.74 \pm$ $0.31 \mathrm{mM}$ compared to control subjects $(1.60 \pm 0.10 \mathrm{mM})^{28}$. This study suggested that the magnitude of lactate elevation may serve as a predictive indicator of morbidity or mortality because patients with poor outcome had significantly higher CSF lactate levels $(5.36 \pm 0.58$ $\mathrm{mM})$ than those from patients who recovered well $(3.01 \pm 0.22 \mathrm{mM})$. However, CSF lactate elevation is common after acute brain injury and might not be specific to convulsive SE ${ }^{29}$. 
Acidosis was also observed in a rat model of SE induced by injection of bicuculline, the $\mathrm{pH}$ falling from $7.41 \pm 0.06$ to $6.91 \pm 0.48$ five minutes after induction of epileptic seizures ${ }^{30}$.

However, there are other sources of acidosis of which muscle contraction. Although lactate is elevated after muscle contraction which is obviously potentially increased after convulsive SE, acidosis is not specifically associated with SE. Nevertheless the correlation between CSF lactate and outcome is promising and acidosis might provide a relevant biomarker of SE.

\section{Acute neuronal injury}

Markers of neuronal injury like Neuron Specific Enolase (NSE), also called enolase-2, could provide an estimate of the degree of brain injury in SE. Enolase is an enzyme expressed in the cytoplasm of all tissues able to perform glycolysis. It catalyzes the conversion of 2-phosphoD-glycerate to phosphoenolpyruvate, and represents a key enzyme of anaerobic glycolysis (a reaction that drives glucose to lactate). Many isoenzymes have been described, depending of which subunits (alpha, beta, gamma) make up the dimers. In the nervous system, glial cells express the $\alpha \alpha$ isoform. Neurons express the NSE, a very stable homodimer of $\gamma$ subunits with a half-life of around 24 hours. It is found in the central and the peripheral nervous system or neuroendocrine tissue, particularly in cells from the APUD system (Amine Precursor Uptake Decarboxylation). Its assay is performed using a monoclonal antibody specific of the $\gamma$ subunit.

NSE levels were first studied in relation to focal or generalized seizures ${ }^{31}$. Patients with frequent seizures or pharmacoresistant seizures showed elevated levels of NSE in both serum and CSF ${ }^{32}$. However, there are conflicting findings for correlation between NSE levels and seizure duration ${ }^{33,34}$.

An increase of CSF concentrations of NSE was first reported in nine of eleven patients with cryptogenic/remote symptomatic SE (without any acute neurologic insult for more than one 
month), compared to twelve control subjects. The mean of CSF-NSE concentrations was higher in SE patients than in control patients $(30.8 \pm 18.33 \text { versus } 10.76 \pm 3.08 \mathrm{ng} / \mathrm{mL})^{35}$. In a prospective study monitoring serum NSE in nineteen patients with sustained SE including eleven without any acute neurologic insult for more than one month, NSE dosages were performed at 24, 48, 72 hours, and seven days after the diagnosis of SE ${ }^{36}$. The mean peak of serum NSE for the entire group $(24.87 \mathrm{ng} / \mathrm{mL})$ and for the eleven patients without an acute neurologic insult (15.44 ng/mL), at 24 and 48 hours after the onset of SE, were increased in comparison to the levels for normal controls $(5.36 \mathrm{ng} / \mathrm{mL})$ and controls with epilepsy (4.61 $\mathrm{ng} / \mathrm{mL}$ ). NSE was normalized at seven days after SE. Moreover, outcome was highly correlated with the peak of serum NSE: serum NSE levels were inversely correlated with the Glasgow Outcome Scale at one week. NSE levels also correlated with the duration of SE: in patients with normal NSE levels at the onset of the SE, SE was significantly shorter than in patients with higher NSE levels ${ }^{36}$. In another study, serum NSE levels were measured in four subtypes of SE: complex partial SE, absence SE, generalized convulsive SE and acute symptomatic myoclonic SE (of which one third is secondary to anoxia) ${ }^{37}$. NSE was significantly elevated in all four subtypes compared to control subjects $(5.02 \mathrm{ng} / \mathrm{mL})$, and NSE levels were higher in complex partial SE $(23.88 \mathrm{ng} / \mathrm{mL})$ and in acute symptomatic myoclonic SE $(37.83 \mathrm{ng} / \mathrm{mL})$. No significant modification in the levels of NSE were found in patients with remote symptomatic SE (i.e. patients without any acute neurologic insult for more than one month, or who had chronic epilepsy or drug withdrawal) when compared with acute symptomatic SE (i.e. patients with any neurologic insult within one month including anoxia) ${ }^{37}$. The highest level of NSE in acute symptomatic myoclonic SE was correlated with the worst outcome, in accordance to the Glasgow Outcome Scale.

Similar results were observed in rats with a SE induced by lithium pilocarpine. In this model, serum NSE levels increased from $5.4 \pm 0.4 \mathrm{ng} / \mathrm{mL}$ in the control condition to $30.4 \pm 1.3$ 
$\mathrm{ng} / \mathrm{mL}$ after SE. Elevation of serum NSE levels after SE correlated with overall histologic evidence for brain damage ${ }^{21}$.

Elevated levels of NSE in the serum may be explained by an increased permeability of the blood-brain barrier ${ }^{35}$. NSE has a quite good neuronal specificity - except in tumoral context and seems to be correlated with the duration and the outcome of SE, two critical requirements for a valid prognosis biomarker. Nevertheless, two antiepileptic drugs commonly used in clinical practice (i.e. carbamazepine and oxcarbazepine) could decrease serum NSE levels ${ }^{38}$. Moreover, NSE is highly sensitive to haemolysis in peripheral blood as well as in CSF. Indeed, NSE levels are markedly increased by in vitro lysis of erythrocytes and thrombocytes, and an artificial increase of NSE level could be seen since the haemoglobin concentration is up to $47 \mathrm{mg}$ per $\mathrm{dL}^{39}$. An adapted pre-analytical approach is needed to avoid haemolysis for a good monitoring of this biomarker.

Therefore, despite good characteristics (good neuronal specificity, stability in the body fluid, links to cellular alterations that occur in SE (i.e. neuronal death) and a good correlation with the duration and outcome of SE), NSE is not currently used as a validated biomarker of SE.

\section{Blood-brain barrier integrity}

The blood-brain barrier (BBB) is the most important vascular barrier of the central nervous system (CNS). It is constituted by a specialized vascular endothelium that interacts with astrocytes, neurons and pericytes and limits penetration of a variety of substances from the blood into the brain. An increased number of spontaneous seizures in pilocarpine-exposed rats was observed in rats with greater BBB dysfunction, suggesting a potential direct role of BBB dysfunction in epileptogenesis ${ }^{40}$. BBB dysfunction can be measured by the CSF/serum albumin ratio ${ }^{41}$. Albumin is mainly synthetized in the liver; therefore, most of albumin in CSF is derived from blood via passage across the BBB. An increase of the CSF/serum 
albumin ratio, indicating BBB damage, is found in patients with various CNS disorders, such as inflammatory diseases, brain tumours or traumatic brain injury.

An increase of the CSF/serum albumin ratio was reported in eleven patients with SE (33.4 $\left.\mathrm{x} 10^{-3}\right)$ compared to twenty control patients $\left(4.79 \times 10^{-3}\right)^{35}$. Higher CSF/serum albumin ratio were found for thirty-one patients with generalized tonic-clonic seizures compared to twentyfive patients without seizure $(8.4 \pm 2.6 \text { versus } 4.7 \pm 1.4)^{42}$.

\section{Acute astroglial injury}

The leakage of serum proteins in the brain due to a BBB dysfunction could lead to a glial activation and inflammation. In SE, increased expression of markers of glial cells (glial fibrillary acidic protein) and proteins produced by glial cells (e.g. cytokines, chemokines, and protein S100-beta also called S100-B) have been reported.

The S100 proteins are a family of calcium-binding proteins with both intracellular and extracellular functions such as the maintenance of cytoskeleton structure, the intracellular communication, the regulation of the cell cycle and energy metabolism and a role in nerve cells growth. The S100 proteins are composed of two subunits alpha and beta, forming dimeric structures, i.e. $\alpha \alpha, \alpha \beta$ or $\beta \beta$. The protein S100-B has at least one beta subunit. S100-B was previously thought to be specific to astrocytes and Schwann cells but S100-B was also detected, albeit at low concentrations, in extracerebral cell types (e.g. chondrocytes, adipocytes, melanocytes). S100-B has a renal elimination and a half-life in blood of around 1.5 hours. At nanomolar concentrations, S100-B can enhance survival of neurons during development and stimulate neurite outgrowth in cortical neurons ${ }^{43}$. In comparison, micromolar levels of S100-B have toxic effects by inducing apoptosis and stimulating the expression of proinflammatory cytokines ${ }^{44}$. S100-B was proposed as a biomarker of neurological disorders thanks to its expression in brain and CSF, its low concentrations in 
serum of normal subjects, its possible extravasation in case of BBB disorder and its release from cellular lysis ${ }^{45}$. An increase of CSF and serum S100-B levels was reported in acute brain injury such as in traumatic brain injury in proportionate terms with the severity of injury 46.

S100-B was studied in isolated seizures. We conducted a study in order to evaluate the performance of S100-B and copeptin in addition to clinical variables, in predicting the outcome of patients hospitalized in emergency department following seizures ${ }^{47}$. S100-B serum levels were shown to be higher in patients who experienced unfavourable outcome (death, hospitalization, seizure recurrence, rehospitalization or a return to the emergency department within seven days) in comparison to other patients. Nevertheless, this biomarker did not improve the prediction of poor outcome following seizures ${ }^{47}$. To the best of our knowledge, no other study evaluated S100-B as a biomarker in SE patients.

The dynamic of astroglial alterations was studied in the lithium-pilocarpine rat model of SE. S100-B CSF levels were increased from the first day (during SE) to the late period marked by the occurrence of spontaneous seizures, suggesting astroglial activation after the onset of SE 48. In the serum, S100-B levels were decreased the day after the lithium-pilocarpine induced SE and increased at 14 days, when compared with levels after sham treatment. The increase in serum S100-B levels suggests astroglial activation and long-term changes in BBB.

S100-B is a potential biomarker for SE: it is expressed in brain and CSF, can be released in the blood in case of BBB alterations like in SE and is increased in a rat model of SE and in patients with seizures. Nevertheless, it still has to be evaluated in patients with SE.

Glial fibrillary acidic protein (GFAP) is a CNS-specific intermediate filament that is exclusively expressed in astrocytes located in the white matter. In the CNS, GFAP is of paramount importance for the structural organisation of astrocytes, the communication 
between astrocytes and some neurons types, including Purkinje cells, and in maintaining the integrity of the BBB.

Elevated CSF GFAP levels were shown in children with seizures as compared with controls 49. In addition, seizure duration was positively correlated with CSF GFAP levels along with SE severity. On the other hand, the aetiologies of the seizures did not seem to affect GFAP level. To the best of our knowledge, no other study in children or adults was conducted. In blood, GFAP might be a better biomarker of SE than S100-B because extracerebral production of GFAP was never detected. Immunohistochemistry studies in animal models of SE have shown increased progressing GFAP staining after SE in hippocampus, substantia nigra and cortical areas ${ }^{50}$.

To conclude, based on the available studies conducted in animal model of SE and in patients with epilepsy, S100-B and GFAP have the potential to be relevant biomarkers of SE due to their expression in brain and CSF, their ability to be released in the blood and their increase after SE. Nevertheless, additional studies on a large cohort of patients with SE with longitudinal follow-up are necessary to better evaluate the capacity of these biomarkers to identify patients with SE and to predict their clinical outcome.

\section{Neuroinflammation}

There is increasing evidence for the role of brain inflammation in epilepsy. Glial cells activation secondary to the $\mathrm{BBB}$ breakdown or neuronal death can induce neuroinflammation and can be followed by the increase of cytokines/chemokines in CSF and in blood of patients with SE. Cytokines such as IL-1ß, IL-6, CRP and the High Mobility Group Box 1 (HMGB1) were measured to investigate their potential biomarker interest in epilepsy. 
HMGB1 fulfils many criteria for a good fluid biomarker, including a good stability in blood. Higher HMGB1 staining was found in mice astrocytes after intrahippocampal injection of kainic acid and in humans epileptic tissues obtained from hippocampal sclerosis surgery ${ }^{51}$. In addition, antagonists of HMGB1 increase delay before the first spontaneous seizure and decrease seizure recurrence ${ }^{51}$. It has to be evaluated in human body fluids too.

Conflicting results were observed for IL-1ß. Interleukin-1 receptor (IL-1R) and its ligand, the pro-inflammatory cytokine IL-1ß are often expressed in association with BBB breakdown and neuronal damage suggesting a key role for the BBB in the brain inflammation observed in both mice and humans with epilepsy ${ }^{25}$. An increase of IL-1ra, the interleukin-1 receptor antagonist, and a decrease of IL-1ß levels were reported in the CSF of eight patients after prolonged focal or recurrent tonic-clonic seizures in comparison to sixteen control subjects ${ }^{52}$. Increased ${ }^{53}$, or decreased blood IL-1ra levels were also reported in patients after seizures ${ }^{54}$. Furthermore, a study conducted in 85 children with generalized tonic-clonic seizures reported a positive correlation between IL-1ß and NSE levels in CSF ${ }^{55}$. To the best of our knowledge, no other study evaluated IL-1ß as a biomarker in SE patients.

In an animal model of SE, an induction of IL-1R and IL-1ß was shown in brain cells following SE 56,57 . For instance, a rapid and persistent increase of IL-1ß mRNA was observed in the hippocampus of a rat model of SE. IL-1ß is a less stable molecule explaining why time that elapses between the seizure occurrence and the samples could play a major role in the variable results described so far.

IL-6 seems to be a good fluid biomarker showing an increase of blood levels described in all studies conducted in patients with epilepsy and in animal models of SE ${ }^{53,58}$. Increased CSF IL-6 was also observed after recurrent generalized tonic-clonic seizures in patients ${ }^{58}$. However, there is a lack of studies conducted in patients regarding the cytokines levels in blood or CSF during SE. 
An acute inflammatory response occurs within the SE. Nevertheless, to the best of our knowledge, the disturbances in cytokines levels and the correlation between the blood cytokines or HMGB1 levels and the patients outcome after SE was not investigated.

\section{Acute axonal injury}

The two best-established CSF biomarkers of axonal injury are tau protein, a phosphorylated microtubule-associated protein, and neurofilament light polypeptide (NFL).

Only few studies evaluated the potential of tau protein as a prognosis biomarker of seizurerelated neuronal damage ${ }^{59}$. CSF levels of tau protein were evaluated in patients with SE and correlated with prognosis ${ }^{60}$. Out of twenty-eight patients with SE, without acute brain injury, CNS infection or neurodegenerative disorders, CSF tau levels were increased in fourteen patients. In addition, higher CSF tau levels were measured in patients who developed a refractory SE compared to patients with SE controlled by antiepileptic drugs. Moreover, CSF tau levels were positively correlated with SE duration and higher risk of sequels and subsequent epilepsy. Nevertheless, CSF tau levels were significantly higher in patients who

were treated by propofol, which is known to induce tau phosphorylation ${ }^{61}$. The marked increase of tau protein in refractory and super-refractory SE cases, and the capacity of tau protein to prospectively identify patients with status severity, development of disability and subsequent epilepsy deserve future verifications.

Neurofilaments are composed of neuron-specific intermediate filaments. Each filament consists of one light subunit (NFL) and a medium subunit (NFM) or a heavy subunit (NFH). High levels of NFH were noted in patients with SE and repetitive generalized tonic clonic seizures in comparison to control subjects ${ }^{62}$. However, no study correlated NFH levels with the clinical outcome of patients. 


\section{Neurogenesis}

Fluid biomarkers may reflect the SE severity as well as neuroprotective mechanisms resulting from the brain adaptation to excitotoxicity. Nevertheless, the current knowledge about neuroprotective mechanisms in humans after SE is scarce. Progranulin (PGRN) is responsible for neurite outgrowth and limits exceeding neuroinflammatory responses. Growing evidence suggests a role for PGRN in the lysosome.

In patients, increased levels of PGRN were measured in the CSF after SE and after a single tonic-clonic seizure, but no significant difference was observed between the two groups ${ }^{24}$. No correlation between progranulin levels and the time of SE was found. Additionally, higher CSF PGRN levels had no impact on clinical outcome. Nevertheless, the cohort was heterogeneous and not fully sufficient. A larger cohort with more homogenous patient is needed to further investigate the relationship between SE and PGRN.

The role of PGRN was investigated in rats after pilocarpine-induced SE ${ }^{63}$. An increase of mRNA and the protein is reported in the cortex and hippocampus. The increase of PGRN was delayed and peaked 48h-96h after the SE onset. PGRN elevation occurs primarily in activated microglia that could explain the elevation delay. Raising cerebral PGRN levels may contribute to neuroprotection by activating endolysosomal system and facilitating regeneration. PGRN could therefore be a potentially new therapeutic strategy in SE.

Neurotrophic factors, involved in the survival of neurons as well as in the proliferation and differentiation of neuronal precursors, could be useful as prognosis biomarkers in SE. An upregulation of neurotrophic factors after SE is seen as reflecting a potential neuroprotective role ${ }^{64}$. Moreover, it was shown that FGF-2/BDNF expressing vectors in pilocarpine model of SE were associated with an increased neurogenesis, limited neuronal damage and reduced occurrence of spontaneous seizures ${ }^{65}$. 


\section{Other potential biomarkers}

This review reports a number of potential protein biomarkers in CSF and blood that were reported in SE. Since a few years, other types of fluid biomarkers are studied.

CSF microRNAs are promising biomarkers of temporal lobe epilepsy and SE; 20 microRNAs showed differential expression between patients in SE and controls patients ${ }^{66}$. Targets of these microRNAs include proteins regulating neuronal death, neuroinflammation, or gliosis; such mechanisms are involved in the late consequences of SE. Therefore, this new approach can be used to distinguish SE from other neurological diseases.

Lipids could provide a new source of biomarkers in SE as well. Indeed, disturbances in brain cholesterol homeostasis were reported in neurological disorders where excitotoxicity plays an important role ${ }^{67}$. Moreover, the accumulation of intracerebral cholesterol is associated with neuronal loss and epileptic abnormalities ${ }^{50}$. The availability of lipid biomarkers measurable at the level of the brain as well as in the serum or the CSF may represent new tools for prospective prognosis studies. Endogenous neurosteroids were studied in SE. They can modulate the excitability of neurons through effects on the $\mathrm{GABA}_{\mathrm{A}}$ receptor chloride channel complex. Two recent studies reported low levels of neurosteroids (i.e. allopregnanolone and progesterone) in CSF of SE patients, suggesting their possible use as a novel therapeutic agent 68,69. The first-in-man allopregnanolone use in adult super-refractory SE provided proof of principle to support its clinical development as a novel therapeutic agent ${ }^{70}$. Nevertheless, a phase 2 trial failed to demonstrate efficacy ${ }^{71}$.

Some biomarkers are more specific to one clinical subtype of SE or aetiology. Therefore, it could be useful to determine specific biomarkers of new-onset refractory status epilepticus (NORSE), that is the most severe form of SE. 


\section{Conclusion}

A large number of biomarkers related to SE reflecting injury of different cell types or structures within the CNS can be measured in CSF and blood. This review summarizes findings of animal and human studies, which were conducted to identify diagnosis and prognosis biomarkers of SE.

Diagnosis of convulsive SE relies mainly on clinical assessment. On the other hand, the diagnosis of non-convulsive SE relies on EEG recording. In this condition, non-EEG biomarkers, like neuronal or axonal injury proteins, could be of interest for the diagnosis and management of non-convulsive SE.

Biomarkers could also be useful to identify the aetiology of SE. Increased NSE levels is further specific of clinical subtypes than for SE aetiology. On the other hand, early increase of cytokines levels, such as IL-1ß, IL-6 or S100-B, should be more frequently associated to infectious or auto-immune SE. These biomarkers could guide the initiation of specific treatment.

Outcome scores based on demographic, clinical and EEG condition are already available. However, they mostly predict the mortality, while the severity of brain injury in survivors is poorly understood. Highest levels of NSE, tau protein and GFAP were correlated to the worst outcome. Elevated levels of serum NSE after generalized tonic-clonic seizures and SE could be primarily due to an increased diffusion of NSE across a more permeable blood-brain barrier, causing a transfer from the brain compartment to the serum compartment. Serum NSE might also be a relevant biomarker of seizures, independent of neuronal death. Otherwise, this explanation would not account for the increase of NSE in the CSF.

Further studies with longitudinal follow-up of these biomarkers and clinical data are needed to evaluate their prognosis value, in particular their ability to prospectively identify patients developing subsequent epilepsy or to prognose the cognitive outcomes. 
To the best of our knowledge, no biomarker described in this review is disease-specific. They were also reported in mild traumatic brain injury (acidosis, NSE, S100-B, cytokines, albumin $\mathrm{CSF} /$ serum ratio), in stroke (NSE, S100-B, cytokines, progranulin), or in infection for cytokines ${ }^{8,72,73}$. A good fluid biomarker is a molecule whose measurement in fluid provides highly sensitive and specific results. A compromise should be defined between the performance of specificity and sensitivity. This could be represented in receiving operator characteristics (ROC) curves to appraise the value of the biomarker and to identify the thresholds (Fig. 4, according to Freund et al. ${ }^{47}$ ). No study of biomarkers in SE reported ROC curves in order to identify their performances. Moreover, among the reports of biomarkers of interest, only one was conducted in accordance with guidelines to uniformly report studies of body fluid biomarker in neurologic disorders ${ }^{74}$. Nevertheless, this study aimed to establish the incremental prognosis value of S100-B after seizures, and so did not concern $\mathrm{SE}^{47}$. According to the Simon's classification, other studies had also the lowest levels of evidence that might be used to determine the clinical utility of a biomarker due to an inappropriate design ${ }^{75}$. We therefore cannot rank the different biomarkers in terms of performance. More studies following these guidelines are needed to validate their clinical potential. We also recommend also conducting separate studies with a specific design in order to evaluate respectively biomarkers for SE diagnosis, for specific aetiology or for prognosis.

A good fluid biomarker is a molecule whose biochemical assessment is easy, quick and reliable and which is stable in the body fluids. We can appraise these different biomarkers on the practicality of measuring. Firstly, we can range biomarkers described above in three categories according to the mean duration of biochemical assessment: hours for acidosis biomarkers, S100-B and NSE; days for albumin ratio and cytokines levels and up to several weeks for progranulin and tau proteins. Secondly, the reliability of the biochemical 
assessment should be taken into account. Data may be more reliable with an automatic method, used in the measurement of S100-B and NSE levels, compared with manual methods, used for the evaluation of progranulin or tau levels. Nevertheless, NSE is highly sensitive to haemolysis and an adapted pre-analytic approach is needed for a good monitoring. Thirdly, some biomarkers are less stable in body fluids. For example, IL-1ß is a less stable molecule when compared with IL-6 and S100-B protein has a shorter half-life compared with NSE, explaining why time that elapses between the seizure occurrence and the samples could induce variable results. Based on their physical and biochemical characteristics, NSE seems to be the best valuable biomarker.

Identification of suitable biomarkers could help clinicians establish a more accurate diagnosis, in particular for the non-convulsive type of SE, to identify SE aetiology, to predict clinical outcome and to manage the treatments. Biomarkers would be combined with clinical and paraclinical data (EEG and MRI) for an overall view of a patient admitted in an intensive care unit with SE.

\section{Key Points}

- Refractory status epilepticus is associated with molecular and cellular changes that may induce brain injury with neuronal loss and subsequent epilepsy

- Specific biomarkers can be detected in the fluids and the brain tissues obtained from patients and animal models of status epilepticus

- Neuron Specific Enolase protein is a useful biomarker of the neuronal loss for which highest levels were correlated with the worst outcome

- Elevated cytokines levels could suggest infectious or auto-immune status epilpeticus and identify specific etiologies requiring individualized treatments 
- Further studies with longitudinal follow-up of these biomarkers and clinical data are needed to better evaluate their prognosis value in status epilepticus

\section{Acknowledgments}

The authors thank Stephen Withmarsh and Mark Williams (Institut du Cerveau et de la Moelle épinière, ICM, Inserm U 1127, CNRS UMR 7225, Sorbonne Université, F-75013, Paris, France) for critical reading of the manuscript.

This work received support from the "Investissements d'avenir" program ANR-10-IAIHU-06, from the "Fondation pour la Recherche Médicale" (FDM20170839111) and from the Fondation pour la Recherche de l'Assitance Publique-Hôpitaux de Paris (EPIRES- MarieLaure PLV Merchandising ).

\section{Disclosures of Conflicts of Interest}

V.N. reports personal fees from UCB, Liva Nova, and EISAI, outside the submitted work. Others authors report no disclosures.

\section{Ethical Publication Statement}

We confirm that we have read the Journal's position on issues involved in ethical publication and affirm that this report is consistent with those guidelines 


\section{References}

1. Fiest KM, Sauro KM, Wiebe S, et al. Prevalence and incidence of epilepsy: A systematic review and meta-analysis of international studies. Neurology. 2017; 88:296-303.

2. Trinka E, Cock H, Hesdorffer D, et al. A definition and classification of status epilepticus--Report of the ILAE Task Force on Classification of Status Epilepticus. Epilepsia. 2015; 56:1515-23.

3. Rossetti AO, Lowenstein DH. Management of refractory status epilepticus in adults: still more questions than answers. Lancet Neurol. 2011; 10:922-30.

4. Rossetti AO, Logroscino G, Bromfield EB. A clinical score for prognosis of status epilepticus in adults. Neurology. 2006; 66:1736-8.

5. Leitinger M, Trinka E, Giovannini G, et al. Epidemiology of status epilepticus in adults: A population-based study on incidence, causes, and outcomes. Epilepsia. 2019; 60:5362.

6. Meletti S, Giovannini G, d'Orsi G, et al. New-Onset Refractory Status Epilepticus with Claustrum Damage: Definition of the Clinical and Neuroimaging Features. Front Neurol. $2017 ; 8: 111$.

7. Tschampa HJ, Greschus S, Sassen R, et al. Thalamus lesions in chronic and acute seizure disorders. Neuroradiology. 2011; 53:245-54.

8. Zetterberg H, Smith DH, Blennow K. Biomarkers of mild traumatic brain injury in cerebrospinal fluid and blood. Nat Rev Neurol. 2013; 9:201-10.

9. Blennow K, Hampel H, Weiner M, et al. Cerebrospinal fluid and plasma biomarkers in Alzheimer disease. Nat Rev Neurol. 2010; 6:131-44. 
10. Hall S, Surova Y, Öhrfelt A, et al. CSF biomarkers and clinical progression of Parkinson disease. Neurology. 2015; 84:57-63.

11. Link H, Huang Y-M. Oligoclonal bands in multiple sclerosis cerebrospinal fluid: an update on methodology and clinical usefulness. J Neuroimmunol. 2006; 180:17-28.

12. Trinka E, Kälviäinen R. 25 years of advances in the definition, classification and treatment of status epilepticus. Seizure. 2017; 44:65-73.

13. Ciurans J, Grau-López L, Jiménez M, et al. Refractory status epilepticus: Impact of baseline comorbidity and usefulness of STESS and EMSE scoring systems in predicting mortality and functional outcome. Seizure. 2018; 56:98-103.

14. Alvarez V, Westover MB, Drislane FW, et al. Evaluation of a clinical tool for early etiology identification in status epilepticus. Epilepsia. 2014; 55:2059-68.

15. Jun J-S, Lee S-T, Kim R, et al. Tocilizumab treatment for new onset refractory status epilepticus. Ann Neurol. 2018; 84:940-5.

16. Meldrum BS, Horton RW. Physiology of status epilepticus in primates. Arch Neurol. $1973 ; 28: 1-9$.

17. Huang C-W, Cheng J-T, Tsai J-J, et al. Diabetic hyperglycemia aggravates seizures and status epilepticus-induced hippocampal damage. Neurotox Res. 2009; 15:71-81.

18. Sutter R, Dittrich T, Semmlack S, et al. Acute Systemic Complications of Convulsive Status Epilepticus-A Systematic Review. Crit Care Med. 2018; 46:138-45.

19. Jutila L, Immonen A, Partanen K, et al. Neurobiology of epileptogenesis in the temporal lobe. Adv Tech Stand Neurosurg. 2002; 27:5-22. 
20. Brandt C, Gastens AM, Sun M zhen, et al. Treatment with valproate after status epilepticus: effect on neuronal damage, epileptogenesis, and behavioral alterations in rats. Neuropharmacology. 2006; 51:789-804.

21. Sankar R, Shin DH, Wasterlain CG. Serum neuron-specific enolase is a marker for neuronal damage following status epilepticus in the rat. Epilepsy Res. 1997; 28:129-36.

22. Parent JM, Yu TW, Leibowitz RT, et al. Dentate granule cell neurogenesis is increased by seizures and contributes to aberrant network reorganization in the adult rat hippocampus. J Neurosci Off J Soc Neurosci. 1997; 17:3727-38.

23. Jakubs K, Nanobashvili A, Bonde S, et al. Environment matters: synaptic properties of neurons born in the epileptic adult brain develop to reduce excitability. Neuron. 2006; $52: 1047-59$.

24. Huchtemann T, Körtvélyessy P, Feistner H, et al. Progranulin levels in status epilepticus as a marker of neuronal recovery and neuroprotection. Epilepsy Behav. 2015; 49:170-2.

25. Ravizza T, Gagliardi B, Noé F, et al. Innate and adaptive immunity during epileptogenesis and spontaneous seizures: evidence from experimental models and human temporal lobe epilepsy. Neurobiol Dis. 2008; 29:142-60.

26. Vezzani A, Baram TZ. New roles for interleukin-1 Beta in the mechanisms of epilepsy. Epilepsy Curr. 2007; 7:45-50.

27. Gorter JA, van Vliet EA, Aronica E. Status epilepticus, blood-brain barrier disruption, inflammation, and epileptogenesis. Epilepsy Behav. 2015; 49:13-6.

28. Calabrese VP, Gruemer HD, James K, et al. Cerebrospinal fluid lactate levels and prognosis in status epilepticus. Epilepsia. 1991; 32:816-21. 
29. Chatzikonstantinou A, Ebert AD, Hennerici MG. Cerebrospinal fluid findings after epileptic seizures. Epileptic Disord Int Epilepsy J Videotape. 2015; 17:453-9.

30. Sokrab TE, Kalimo H, Johansson BB. Endogenous serum albumin content in brain after short-lasting epileptic seizures. Brain Res. 1989; 489:231-6.

31. Royds JA, Davies-Jones GA, Lewtas NA, et al. Enolase isoenzymes in the cerebrospinal fluid of patients with diseases of the nervous system. J Neurol Neurosurg Psychiatry. $1983 ; 46: 1031-6$.

32. Rabinowicz AL, Correale J, Boutros RB, et al. Neuron-specific enolase is increased after single seizures during inpatient video/EEG monitoring. Epilepsia. 1996; 37:122-5.

33. Suzuki Y, Toribe Y, Goto M, et al. Serum and CSF neuron-specific enolase in patients with West syndrome. Neurology. 1999; 53:1761-4.

34. Tanabe T, Suzuki S, Hara K, et al. Cerebrospinal fluid and serum neuron-specific enolase levels after febrile seizures. Epilepsia. 2001; 42:504-7.

35. Correale J, Rabinowicz AL, Heck CN, et al. Status epilepticus increases CSF levels of neuron-specific enolase and alters the blood-brain barrier. Neurology. 1998; 50:138891.

36. DeGiorgio CM, Correale JD, Gott PS, et al. Serum neuron-specific enolase in human status epilepticus. Neurology. 1995; 45:1134-7.

37. DeGiorgio CM, Heck CN, Rabinowicz AL, et al. Serum neuron-specific enolase in the major subtypes of status epilepticus. Neurology. 1999; 52:746-9. 
38. Maiti R, Mishra BR, Sanyal S, et al. Effect of carbamazepine and oxcarbazepine on serum neuron-specific enolase in focal seizures: A randomized controlled trial. Epilepsy Res. $2017 ; 138: 5-10$.

39. Ramont L, Thoannes H, Volondat A, et al. Effects of hemolysis and storage condition on neuron-specific enolase (NSE) in cerebrospinal fluid and serum: implications in clinical practice. Clin Chem Lab Med. 2005; 43:1215-7.

40. van Vliet EA, da Costa Araújo S, Redeker S, et al. Blood-brain barrier leakage may lead to progression of temporal lobe epilepsy. Brain J Neurol. 2007; 130:521-34.

41. Tibbling G, Link H, Ohman S. Principles of albumin and IgG analyses in neurological disorders. I. Establishment of reference values. Scand J Clin Lab Invest. 1977; 37:38590.

42. Li Y-J, Wang Z-H, Zhang B, et al. Disruption of the blood-brain barrier after generalized tonic-clonic seizures correlates with cerebrospinal fluid MMP-9 levels. J Neuroinflammation. 2013; 10:80.

43. Donato R. S100: a multigenic family of calcium-modulated proteins of the EF-hand type with intracellular and extracellular functional roles. Int J Biochem Cell Biol. 2001; 33:637-68.

44. Sen J, Belli A. S100B in neuropathologic states: the CRP of the brain? J Neurosci Res. $2007 ; 85: 1373-80$.

45. Beaudeux J-L, Laribi S. [S100B protein serum level as a biomarker of minor head injury]. Ann Biol Clin (Paris). 2013; 71:71-8. 
46. Rezaei O, Pakdaman H, Gharehgozli K, et al. S100 B: A new concept in neurocritical care. Iran J Neurol. 2017; 16:83-9.

47. Freund Y, Bloom B, Bokobza J, et al. Predictive value of S100-B and copeptin for outcomes following seizure: the BISTRO International Cohort Study. PloS One. 2015; 10:e0122405.

48. Vizuete AFK, Hennemann MM, Gonçalves CA, et al. Phase-Dependent Astroglial Alterations in Li-Pilocarpine-Induced Status Epilepticus in Young Rats. Neurochem Res. 2017; 42:2730-42.

49. Gurnett CA, Landt M, Wong M. Analysis of cerebrospinal fluid glial fibrillary acidic protein after seizures in children. Epilepsia. 2003; 44:1455-8.

50. Chali F, Djelti F, Eugene E, et al. Inhibiting cholesterol degradation induces neuronal sclerosis and epileptic activity in mouse hippocampus. Eur J Neurosci. 2015; 41:134555.

51. Maroso M, Balosso S, Ravizza T, et al. Toll-like receptor 4 and high-mobility group box1 are involved in ictogenesis and can be targeted to reduce seizures. Nat Med. 2010; $16: 413-9$.

52. Lehtimäki KA, Keränen $T$, Palmio J, et al. Levels of IL-1-beta; and IL-1ra in Cerebrospinal Fluid of Human Patients after Single and Prolonged Seizures. Neuroimmunomodulation. 2010; 17:19-22.

53. Lehtimäki KA, Keränen T, Palmio J, et al. Increased plasma levels of cytokines after seizures in localization-related epilepsy. Acta Neurol Scand. 2007; 116:226-30. 
54. Alapirtti T, Rinta S, Hulkkonen J, et al. Interleukin-6, interleukin-1 receptor antagonist and interleukin-1beta production in patients with focal epilepsy: A video-EEG study. $\mathrm{J}$ Neurol Sci. 2009; 280:94-7.

55. Shi L-M, Chen R-J, Zhang H, et al. Cerebrospinal fluid neuron specific enolase, interleukin- $1 \beta$ and erythropoietin concentrations in children after seizures. Childs Nerv Syst ChNS Off J Int Soc Pediatr Neurosurg. 2017; 33:805-11.

56. Lehtimäki KA, Peltola J, Koskikallio E, et al. Expression of cytokines and cytokine receptors in the rat brain after kainic acid-induced seizures. Brain Res Mol Brain Res. $2003 ; 110: 253-60$.

57. Vezzani A, Conti M, De Luigi A, et al. Interleukin-1beta immunoreactivity and microglia are enhanced in the rat hippocampus by focal kainate application: functional evidence for enhancement of electrographic seizures. J Neurosci Off J Soc Neurosci. 1999; 19:5054-65.

58. Lehtimäki KA, Keränen T, Huhtala H, et al. Regulation of IL-6 system in cerebrospinal fluid and serum compartments by seizures: the effect of seizure type and duration. $\mathrm{J}$ Neuroimmunol. 2004; 152:121-5.

59. Palmio J, Suhonen J, Keränen T, et al. Cerebrospinal fluid tau as a marker of neuronal damage after epileptic seizure. Seizure. 2009; 18:474-7.

60. Monti G, Tondelli M, Giovannini G, et al. Cerebrospinal fluid tau proteins in status epilepticus. Epilepsy Behav EB. 2015; 49:150-4. 
61. Whittington RA, Bretteville A, Virág L, et al. Anesthesia-induced hypothermia mediates decreased ARC gene and protein expression through ERK/MAPK inactivation. Sci Rep. $2013 ; 3: 1388$.

62. Rejdak K, Kuhle J, Rüegg S, et al. Neurofilament heavy chain and heat shock protein 70 as markers of seizure-related brain injury. Epilepsia. 2012; 53:922-7.

63. Zhu S, Tai C, Petkau TL, et al. Progranulin promotes activation of microglia/macrophage after pilocarpine-induced status epilepticus. Brain Res. 2013; 1530:54-65.

64. Lenzer-Fanara JR, Li T, Salerni EA, et al. VEGF treatment during status epilepticus attenuates long-term seizure-associated alterations in astrocyte morphology. Epilepsy Behav EB. 2017; 70:33-44.

65. Simonato M, Zucchini S. Are the neurotrophic factors a suitable therapeutic target for the prevention of epileptogenesis? Epilepsia. 2010; 51:48-51.

66. Raoof R, Jimenez-Mateos EM, Bauer S, et al. Cerebrospinal fluid microRNAs are potential biomarkers of temporal lobe epilepsy and status epilepticus. Sci Rep. 2017; $7: 3328$.

67. Valenza M, Cattaneo E. Cholesterol dysfunction in neurodegenerative diseases: is Huntington's disease in the list? Prog Neurobiol. 2006; 80:165-76.

68. Meletti S, Lucchi C, Monti G, et al. Decreased allopregnanolone levels in cerebrospinal fluid obtained during status epilepticus. Epilepsia. 2017; 58:e16-20.

69. Meletti S, Lucchi C, Monti G, et al. Low levels of progesterone and derivatives in cerebrospinal fluid of patients affected by status epilepticus. J Neurochem. 2018; $147: 275-84$. 
70. Vaitkevicius H, Husain AM, Rosenthal ES, et al. First-in-man allopregnanolone use in super-refractory status epilepticus. Ann Clin Trans1 Neurol. 2017; 4:411-4.

71. Rosenthal ES, Claassen J, Wainwright MS, et al. Brexanolone as adjunctive therapy in super-refractory status epilepticus. Ann Neurol. 2017; 82:342-52.

72. Ng GJL, Quek AML, Cheung C, et al. Stroke biomarkers in clinical practice: A critical appraisal. Neurochem Int. 2017; 107:11-22.

73. van Engelen TSR, Wiersinga WJ, Scicluna BP, et al. Biomarkers in Sepsis. Crit Care Clin. 2018; 34:139-52.

74. Gnanapavan S, Hegen H, Khalil M, et al. Guidelines for uniform reporting of body fluid biomarker studies in neurologic disorders. Neurology. 2014; 83:1210-6.

75. Simon RM, Paik S, Hayes DF. Use of Archived Specimens in Evaluation of Prognostic and Predictive Biomarkers. JNCI J Natl Cancer Inst. 2009; 101:1446-52. 


\section{Figures legends}

Fig. 1: Progressive brain MRI changes in a 17-year-old patient suffering from new-onset prolonged refractory status epilepticus.

MRI was performed a few days after the onset of a generalized status epilepticus (A, C) and after 8 weeks (B, D). Axial FLAIR sequences (A, B) showed an initial hypersignal in the claustrum and the pulvinar (arrows). Coronal T1 sequences (C, D) showed a major atrophy on the second MRI (D), affecting both cortical and sub-cortical regions, i.e enlargement of ventricules and hippocampal atrophy (arrows).

\section{Fig. 2: Major EEG changes during status epilepticus}

Extract of an EEG (A), of a bipolar montage, from the same patient, as in Fig.1, showing long periods of isoelectric activity (first seconds of the trace) related to high doses of barbiturate, that alternate with repetitive seizures starting and ending with high-amplitude spikes. Time frequency analysis (B) of the EEG signal shows the spectral signature of a seizure.

Extract of an EEG (C), of a bipolar montage, from a 20-year-old patient presenting with a refractory status epilepticus related to a NMDAr encephalitis. The EEG shows a continuous waxing and waning of ictal activity.

Time frequency analysis (D) of a longer period (10 minutes) showing the spectral signature of two seizures. The spectral signature of the seizure $\mathrm{C}$ is shown in the dotted box.

Fig. 3: Biomarkers related to the pathophysiology of status epilepticus.

During status epilepticus molecular and cellular changes occur: neuronal death is followed by the release of Neuron Specific Enolase (1) and neuroinflammation with production of cytokines (2). The blood brain barrier is disrupted; albumin may worsen the inflammatory process (3) and leukocytes can be attracted (4). All these processes result in gliosis (5) that 
can be identified by GFAP and S100-B analysis. All these molecules could cross the blood brain barrier and be measured in the blood samples (6).

Abbreviations: BBB, blood brain barrier; GFAP, Glial Fibrillary Acidic Protein; IL, interleukin; NSE, Neuron Specific Enolase.

\section{Fig. 4: Example of a receiving operator characteristic curve for S100-B.}

Reprinted from Predictive Value of S100-B and Copeptin for Outcomes following Seizure: the BISTRO International Cohort Study (p.8), Yonathan Freund, 2015, PLOS One.

This study evaluated the performance of S100-B in addition to clinical variables in predicting outcome of patients following seizure. A receving operator characteristic curve was constructed. It could be used to appraise the value of a biomarker by calculating the area under the curve and to identify thresholds using the Youden's method. A sensitivity of 0.57 and a specificity of 0.53 for S100-B were defined.

The best biomarker would have simultaneously a sensitivity around $100 \%$ and a score " 1 specificity" around zero. 


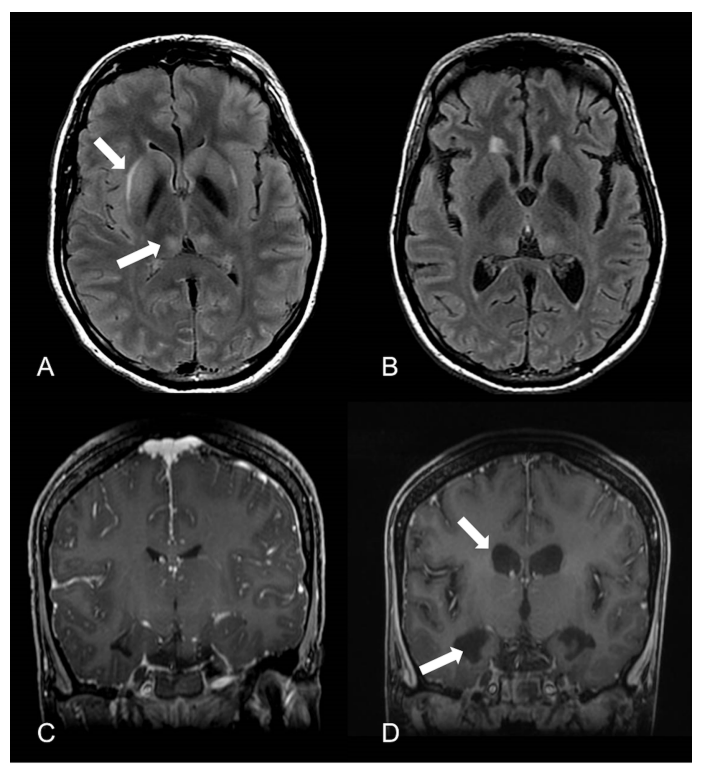




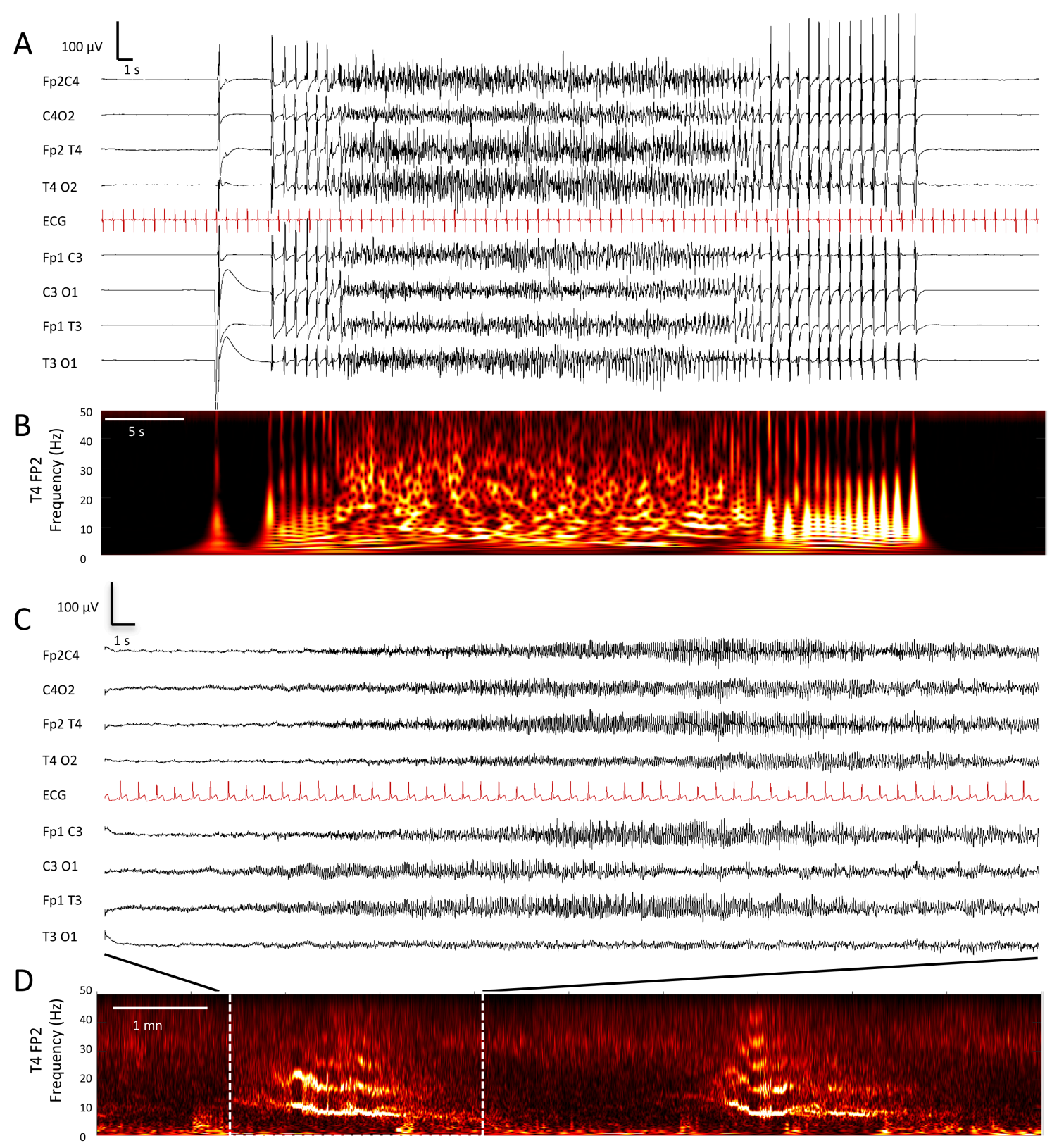




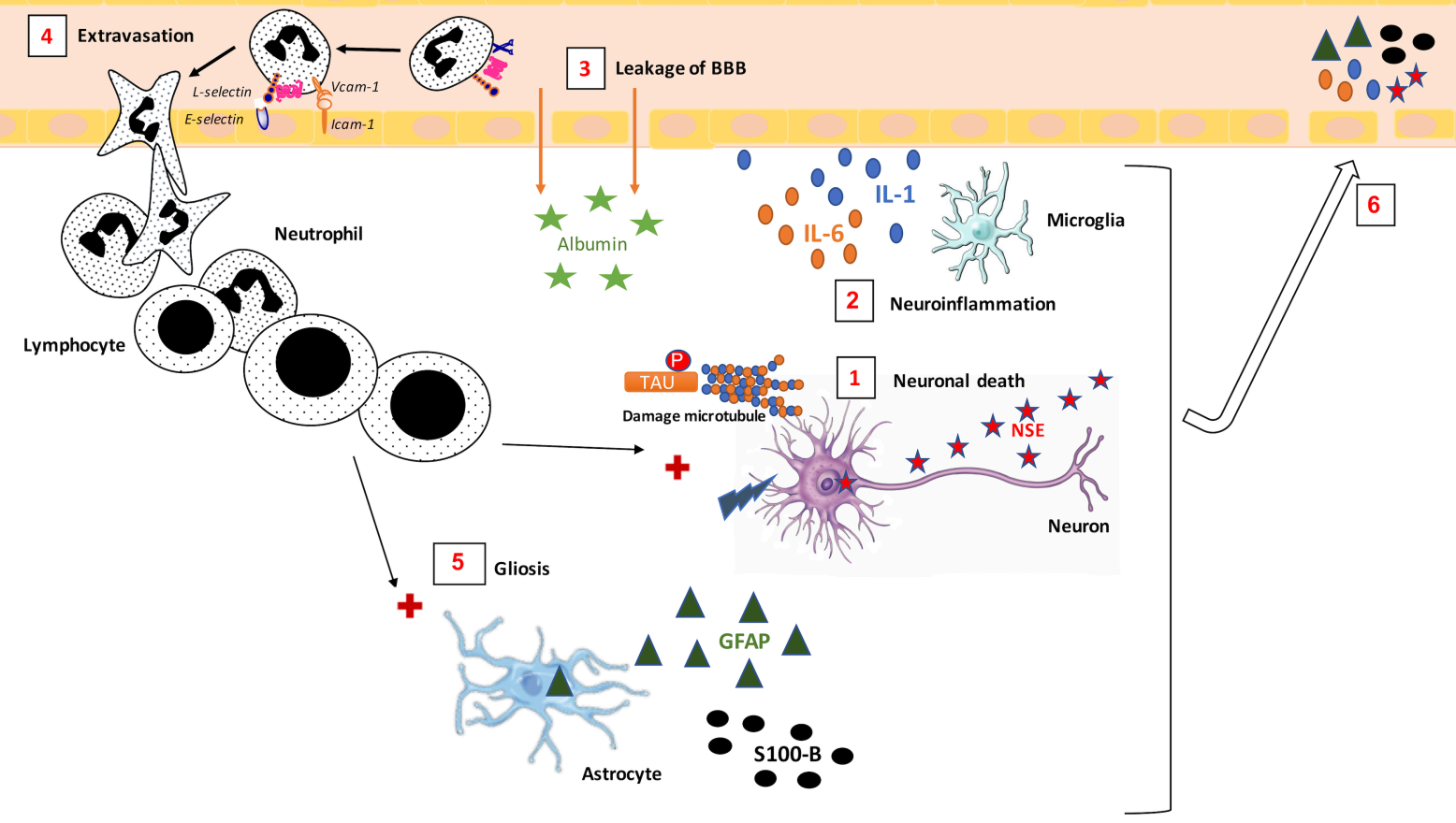




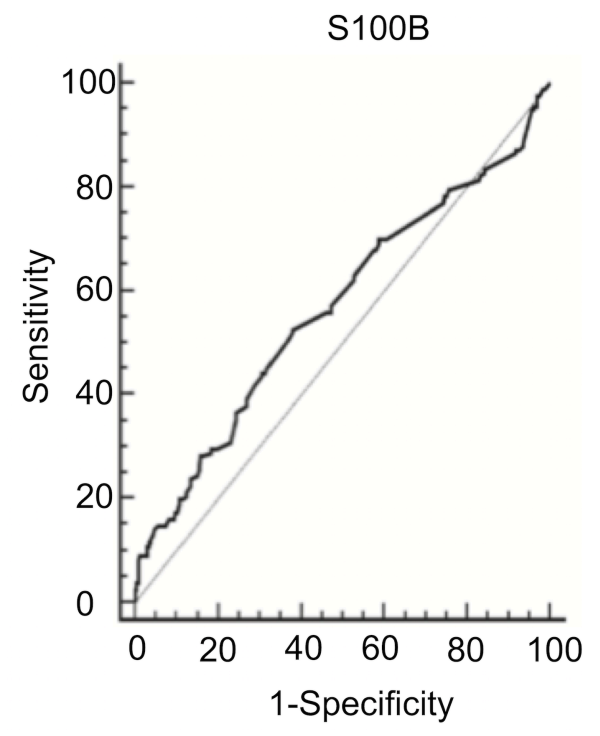




\begin{tabular}{|c|c|c|c|c|c|c|c|c|}
\hline \multirow[t]{2}{*}{ Pathophysiology } & \multirow[t]{2}{*}{ Biomarker } & \multicolumn{2}{|c|}{ Research findings in CSF / brain tissues } & \multicolumn{2}{|c|}{ Research findings in blood } & \multirow[t]{2}{*}{ Prognostic value } & \multirow[t]{2}{*}{ Advantages } & \multirow[t]{2}{*}{ Current limitations } \\
\hline & & Animal models & Humans & Animal models & Humans & & & \\
\hline \multirow[t]{2}{*}{ Acidosis } & Lactate & l & $\begin{array}{c}\text { Levels are elevated in patients } \\
\text { with generalized } \mathrm{SE}^{28}\end{array}$ & l & / & $\begin{array}{c}\text { Magnitude of elevation as } \\
\text { indicator of morbidity or } \\
\text { mortality }\end{array}$ & \multirow{2}{*}{$\begin{array}{l}\text { Quick biochemical } \\
\text { assessment }\end{array}$} & \multirow{2}{*}{$\begin{array}{c}\text { Not specifically } \\
\text { associated to SE } \\
\text { No study performed on } \\
\text { non-convulsive SE }\end{array}$} \\
\hline & pH & l & l & $\begin{array}{l}\mathrm{pH} \text { fell five minutes after the } \\
\text { induction of epileptic seizures }{ }^{30}\end{array}$ & l & / & & \\
\hline $\begin{array}{l}\text { Acute neuronal } \\
\text { injury }\end{array}$ & NSE & l & $\begin{array}{c}\text { Increase of NSE levels in } \\
\text { patients with } \\
\text { cryptogenic/symptomatic } \mathrm{SE}^{35}\end{array}$ & $\begin{array}{c}\text { Elevated levels in a lithium } \\
\text { pilocarpine animal model of } \mathrm{SE}^{21}\end{array}$ & $\begin{array}{c}\text { Levels peak at } 24-48 \text { hours } \\
\text { after the onset of SE, } \\
\text { normalized at } 7 \text { days }^{36} \\
\text { Highest level in subclinical } \\
\text { generalized convulsive } \mathrm{SE}^{37}\end{array}$ & $\begin{array}{c}\text { Outcome highly } \\
\text { correlated with the peak } \\
\text { of serum } \mathrm{NSE}^{36,37}\end{array}$ & $\begin{array}{l}\text { Good neuronal } \\
\text { specificity } \\
\text { High stability in body } \\
\text { fluid } \\
\text { Correlation to neuronal } \\
\text { death, duration and } \\
\text { outcome of SE }\end{array}$ & $\begin{array}{c}\text { Highly sensitivity to } \\
\text { haemolysis } \\
\text { Serum levels could be } \\
\text { decreased by two major } \\
\text { antiepileptic drugs (bias } \\
\text { for prognostic } \\
\text { evaluation) }\end{array}$ \\
\hline $\begin{array}{c}\text { Blood-brain barrier } \\
\text { breakdown }\end{array}$ & $\begin{array}{c}\text { CSF-serum albumin } \\
\text { ratio }\end{array}$ & l & $\begin{array}{l}\text { Increase of the ratio in patients } \\
\text { with SE and generalized tonic- } \\
\text { clonic seizures in comparison } \\
\text { to controls }{ }^{35,42}\end{array}$ & / & l & l & / & \begin{tabular}{|} 
Not specific to SE \\
Lumbar puncture not \\
systematically \\
performed in clinical \\
assessment
\end{tabular} \\
\hline Gliosis & S100-B & $\begin{array}{c}\text { Increase from the first } \\
\text { day to the late period (56 } \\
\text { days) })^{48}\end{array}$ & / & $\begin{array}{l}\text { Decrease of S100-B at one day after } \\
\text { the lithium-pilocarpine injection and } \\
\text { elevation at } 14 \text { days }^{48}\end{array}$ & $\begin{array}{l}\text { Higher S100-B levels in } \\
\text { epileptic patients }^{47}\end{array}$ & $\begin{array}{l}\text { Higher S100-B serum } \\
\text { levels associated with } \\
\text { unfavorable outcome }{ }^{47}\end{array}$ & $\begin{array}{c}\text { Almost brain specific } \\
\text { Release in blood only } \\
\text { in case of BBB } \\
\text { breakdown }\end{array}$ & $\begin{array}{c}\text { No study conducted in } \\
\text { blood or CSF of SE } \\
\text { patients }\end{array}$ \\
\hline
\end{tabular}




\begin{tabular}{|c|c|c|c|c|c|c|c|c|}
\hline & GFAP & $\begin{array}{l}\text { Increase staining in } \\
\text { hippocampus }^{50}\end{array}$ & $\begin{array}{c}\text { Elevated CSF GFAP levels in } \\
\text { children after seizures }{ }^{49}\end{array}$ & l & I & $\begin{array}{l}\text { CSF GFAP correlated } \\
\text { with SE severity }{ }^{49}\end{array}$ & $\begin{array}{c}\text { No extracerebral } \\
\text { production of GFAP }\end{array}$ & $\begin{array}{l}\text { Need more study with } \\
\text { larger cohorts and } \\
\text { longitudinal follow up } \\
\text { to validate its } \\
\text { prognostic interest }\end{array}$ \\
\hline \multirow{3}{*}{ Neuroinflammation } & IL-1B & \begin{tabular}{|l|} 
Increase of Il-1 $13 \mathrm{mRNA}$ \\
in the hippocampus \\
6,57
\end{tabular} & $\begin{array}{c}\text { Increase of IL-1 ra and decrease } \\
\text { of IL- } 1 \beta^{52}\end{array}$ & I & Discordant results ${ }^{53,54}$ & I & l & Unstable molecule \\
\hline & IL-6 & / & l & Increase blood levels in all studies ${ }^{53,58}$ & $\begin{array}{c}\text { Increase blood levels in all } \\
\text { studies }^{53,58}\end{array}$ & I & I & Unstable molecule \\
\hline & HMGB1 & $\begin{array}{c}\text { Increase staining after } \\
\text { kainic acid and in human } \\
\text { epileptic tissues }^{51}\end{array}$ & 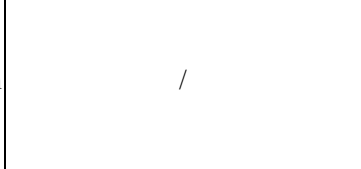 & I & I & I & Good stability in blood & $\begin{array}{c}\text { No study conducted in } \\
\text { human body fluids }\end{array}$ \\
\hline \multirow[t]{2}{*}{ Axonal injury } & Tau & l & $\begin{array}{c}\text { Higher CSF levels in } 50 \% \text { of } \\
\text { patients with } \mathrm{SE}^{60}\end{array}$ & l & I & $\begin{array}{c}\text { Higher CSF tau levels in } \\
\text { patients with refractory } \\
\text { SE, disabilities and } \\
\text { chronic epilepsy }^{60}\end{array}$ & $\begin{array}{c}\text { Correlation to SE } \\
\text { outcome }\end{array}$ & $\begin{array}{c}\text { Influence of anesthesics } \\
\text { drugs } \\
\text { No study conducted in } \\
\text { blood samples }\end{array}$ \\
\hline & NFH & l & $\begin{array}{c}\text { Higher levels in patients with } \\
\text { SE and repetitive generalized } \\
\text { tonic clonic seizures }{ }^{62}\end{array}$ & l & l & l & l & $\begin{array}{c}\text { No study conducted in } \\
\text { blood samples }\end{array}$ \\
\hline Neurogenesis & Progranulin & $\begin{array}{l}\text { Increase of the mRNA in } \\
\text { the hippocampus and } \\
\text { cortex at } 48-96 \text { hours }^{63}\end{array}$ & $\begin{array}{c}\text { Increased levels of PGRN in } \\
\text { patients with } \mathrm{SE}^{24}\end{array}$ & I & I & $\begin{array}{c}\text { No impact on clinical } \\
\text { outcome }^{24}\end{array}$ & I & $\begin{array}{c}\text { No study conducted in } \\
\text { blood samples }\end{array}$ \\
\hline
\end{tabular}


Table 1: Potential CSF and blood biomarkers in status epilepticus.

Summary of clinical and preclinical studies.

Abbreviations: NSE, Neuron Specific Enolase; GFAP, Glial Fibrillary Acidic Protein; HMGB1, High Mobility Group Box 1, NFH, Neurofilament Heavy Chain Protein 